\title{
Recommended standards for modern tuberculosis laboratory services in Europe
}

\author{
F.A. Drobniewski*, S. Hoffner", S. Rusch-Gerdes ", G. Skenders ${ }^{+}$, \\ V. Thomsen ${ }^{\S}$ and the WHO European Laboratory Strengthening Task Force
}

ABSTRACT: The principles underpinning these standards are that any tuberculosis laboratorybased diagnostic procedure should be performed by appropriately trained staff, working to standardised operating procedures in appropriately equipped and safe laboratories, against clear national and international proficiency and quality standards. Quality should be the pre-eminent criteria, not cost.

The standards are technologically feasible, but initially may not be within the financial capacity of all laboratories. There is a requirement for government and international donors to adequately fund an appropriate safe infrastructure to enable staff to deliver accurate and timely results at whatever level of activity they are performing.

There is a need for national reference laboratories to train a new cadre of mycobacterial laboratory experts. This will require the funding of appropriate individuals at these centres to train and assist in the implementation of good laboratory practice and evaluation to build sustainable capacity. Further operational research is needed to establish the optimal configuration of new technologies to determine isoniazid, rifampicin and second-line drug susceptibility in mycobacterial cultures and also, increasingly, directly on specimens.

Improved integration of laboratory medicine as a core part of all tuberculosis programmes is needed to achieve and maximise the potential of new developments.

KEYWORDS: Culture, guidelines, microscopy, molecular testing, training, tuberculosis

$\mathbf{T}$ here were 9 million new tuberculosis (TB) cases and $\sim 2$ million TB deaths in 2004, of which 3.9 million (62 out of 100,000$)$ were smear positive, and 741,000 were in adults infected with HIV [1]. In the most recent World Health Organization (WHO) report on Global Tuberculosis Control, a decade of progress towards achieving the targets proposed by the World Health Assembly and the Millennium Development Goals (MDGs) was summarised. The WHO targets were to detect, by $2005,70 \%$ of new sputum smear-positive cases and to successfully treat $85 \%$ of these cases. The MDG Target 8 (of 18) is to have halted and begun to reverse the TB incidence rate by 2015 . The Stop TB Partnership endorsed additional targets of halving the 1990 prevalence and deaths rates by 2015 .

\section{WHO Euro Laboratory Task Force}

The WHO Laboratory Strengthening Task Force (LSTF) for Tuberculosis Control for the WHO European Region was established in 2005 at the recommendation of the WHO European Technical Advisory Group (TAG). The main function of the LSTF is to provide guidance to the WHO and TAG on strategic and technical aspects of TB laboratory capacities within the WHO European region. The LSTF acts as a technical advisory board, assisting in situational analyses of laboratory services in priority countries, reviewing relevant international laboratory documents and guidelines, assisting the WHO in estimating budgets needed for laboratory capacity strengthening, and helping to identify and train national and international experts to assist countries in strengthening their national $\mathrm{TB}$ services.

The need for such a group was driven by the increasing incidence and prevalence of $\mathrm{TB}$ in Europe as well as globally, particularly in those countries with the highest burdens of $\mathrm{TB}$, and low rates of laboratory case detection and confirmation of TB cases. In 2004, the global case detection rate was $53 \%$, and would exceed $60 \%$ in
AFFILIATIONS

*HPA National Mycobacterium Reference Unit and Clinical TB and HIV Group, London, UK

\#Swedish Institute for Infectious Disease Control, Stockholm, Sweden. "Forschungszentrum Borstel, Borstel, Germany.

+National TB Institute, Riga, Latvia ${ }^{\S}$ Statens Serum Institut, Copenhagen, Denmark.

CORRESPONDENCE

F.A. Drobniewsk

Health Protection Agency National Mycobacterium Reference Unit Head Clinical TB and HIV Group Centre for Infectious Diseases Institute for Cell and Molecular Sciences

Barts and the London School of Medicine

Queen Mary College

2 Newark Street

London

E1 2AT UK

Fax: 442075393459

E-mail: f.drobniewski@qmul.ac.uk

Received:

June 282006

Accepted after revision:

August 012006 
2005 , falling short of the $70 \%$ target. Treatment success was $82 \%$ in the 2003 cohort of 1.7 million patients, approaching the $85 \%$ target [1]. Implementation of the new Global Plan for TB is expected to reverse the rise in incidence globally by 2015, as specified in the MDGs, and to halve the 1990 prevalence and death rates globally and in most regions by 2015, although this is not expected to occur in Africa and Eastern Europe. In the former region this is mainly due to the association with HIV infection, but in Eastern Europe [1,2] the major factor is the high level of drug resistance and particularly multidrugresistant (MDR)-TB [2-6]. This has been due, in large part, to the failure to recognise laboratories as a corner-stone of TB control policy. With this in mind, the current study is aimed at those responsible for managing and implementing TB control programmes as well as heads of laboratories.

The present study is the first paper from the LSTF. It describes the principal standards that a competent TB laboratory, at any level of activity, should aspire to in Europe. Some countries will be in a position to implement these recommended standards sooner than others, but consideration has been given to studies published in the scientific literature and all recommendations are technically feasible. Nevertheless it is not intended to describe in detail how to meet these standards or to present detailed standard operating procedures (SOP). It aims to complement existing WHO and other international recommendations and guidelines [7-23].

\section{KEY PRINCIPLES AND OVERARCHING RECOMMENDATIONS}

The underlying principles underpinning this document are that any TB-related procedure (microscopy, bacterial culture, identification, drug susceptibility testing (DST), molecular diagnosis) should be performed by appropriately trained staff, working to SOPs, in appropriately equipped and safe laboratories against clear national and international proficiency and quality standards. Quality rather than cost should be the pre-eminent criteria.

\section{Accreditation}

All laboratories should conduct a thorough internal quality control programme. Analyses and diagnostic services should be accredited wherever such a scheme exists and laboratories must participate in relevant proficiency schemes. Laboratories should not conduct procedures in which they have failed to demonstrate proficiency through a quality control scheme. Similarly, where a licensing system exists, laboratories should be licensed to perform TB-related microbiological activity.

\section{Biosafety and infrastructure}

Laboratory staff are entitled to work in a safe environment. Staff working with patient specimens and live mycobacterial cultures must operate under appropriate biosafety conditions with adequate infection control measures in place, including staff health checks. There are several documents available which can be used as the basis for development of appropriate safety standards [3, 24-27].

Many laboratories do not meet reasonable safety standards and have too few staff to adequately complete their tasks. As infrastructure and biosafety standards are improved, an inevitable consequence is that fewer laboratories should perform complex procedures, such as DST or molecular diagnosis, than at present.

\section{National reference laboratory}

The LSTF supports the recommendations of the WHO Euro Technical Advisory Group that there should be one designated national reference laboratory (NRL) in each country with a designated head. There must be official recognition by the Ministry of Health (or equivalent body) of the NRL, its leadership and the remaining laboratory structure. The NRL head should participate in meetings with the National TB Control Programme manager (or the person fulfilling the equivalent role) and should have sufficient funding for the tasks required of the NRL. Smaller countries with little or no TB may determine that there is no need for their own NRL, in which case the country should establish links with a national (or supranational) reference laboratory in another country. Conversely, in larger countries with a significant TB case load, large regional centres will be needed to amplify and extend the role of the national reference centre.

\section{Funding}

Just as it is appropriate to ask laboratories to meet the standards described below, it is appropriate for government and TB control programmes to provide sufficient funding for laboratories to perform their activities. In particular, the Ministry of Health (or equivalent body) must take direct responsibility for ensuring that there is an adequate NRL budget for all its designated functions.

Funding should ensure the following. 1) A safe and functioning infrastructure with appropriate and well-maintained equipment, including access to sufficient spare parts to maintain activity [23, 24-27]. The most important items are the presence of appropriately maintained class 1,2 or 3 biological safety cabinets, and where concentration techniques are applied, an effective centrifuge in which biological material is contained, preferably within sealed buckets or at least within a "windshield-type" enclosure. High-quality binocular light or fluorescence microscopes, incubators/rooms and refrigerators freezers capable of maintaining an appropriate temperature are also essential items. 2) Sufficient trained staff to perform the expected workload. 3) Sufficient budgetary control at the laboratory level to ensure service continuity and development by those best placed to achieve it. 4) Sufficient laboratory consumables, including disposable plasticware, single-use glass slides ideally with frosted ends, high-quality smearmicroscopy reagents and pure antibiotic drug reagents, for drug susceptibility testing.

\section{Human resources}

Arguably one of the most neglected areas relates to the need for sufficient adequately trained staff to perform TB laboratory procedures, with an understanding of the staff needed to perform the actual workload. Staff numbers for all laboratories including the NRL should be calculated on the basis of TB incidence, number of tests conducted, quality control and other specialised work that the laboratories conduct. Staff should have clear job descriptions and sufficient training to perform their work correctly. 
There is a need for NRLs to have sufficient human resources to provide national as well as international training, to replace staff who have retired or left laboratory medicine and to increase the number of qualified laboratory staff available to support international TB control. In reality in many areas of Europe, the staff age structure has meant that entire laboratories are frequently staffed by too few individuals, many of whom are too close to retirement age. In other industrialised countries such as the USA, a high proportion of experienced technical staff are also approaching retirement age [28] with similar problems reported by many high TB burden countries [29].

\section{Communication}

Good communication is essential both internally, within the laboratory; and externally, between the laboratory and others, including the reporting of results to the relevant clinical staff, others involved in case management or contact tracing/ outbreak investigations, and the national TB programme and surveillance system as defined in each country. Delays in referring specimens and reporting information can lead to delays in diagnosis, treatment and implementation of infection control [30].

Clinicians must inform the laboratory of relevant patient information. Laboratories should provide information on the performance of their analyses and support the taking of appropriate high-quality specimens to improve the sensitivity of analyses. Clinicians should be informed of results as soon as possible.

\section{Laboratory procedures}

It is recommended that microscopy and culture for diagnosis of new cases is performed prior to the institution of treatment using a limited number of high-quality specimens.

\section{Microscopy}

Smear microscopy should be performed within one working day from the arrival of the specimen in the laboratory. Although tests may be performed quickly, laboratories should also identify and address delays in reporting systems. For diagnostic and infection control purposes, positive results should be reported immediately by telephone, fax or other electronic means, as soon as they are available. Although fluorescent microscopy appears to be more sensitive than conventional Ziehl-Neelsen acid-fast staining [31], its principal purpose is to allow greater specimen throughput per microscopist by reducing the time taken to examine each slide.

Ideally, specimens should be transported to laboratories as quickly as possible with an interval of no longer than 4 days before processing. Specimens should be refrigerated if transportation will take $>48 \mathrm{~h}$.

The presence of acid-fast bacilli in a single sputum specimen (smear positive) may be considered as presumptive diagnosis of TB but awaits definitive identification as TB.

Other key extra-pulmonary specimens, such as cerebrospinal fluid, should also be examined within one working day. Resources should be redeployed where they are currently used for an activity of minimal benefit, e.g. there is little or no value in performing microscopic examination of urine samples (except where there is a strong suspicion of renal TB) and this practice should be discontinued.

Although diagnostic sensitivity may be improved by concentration methods including centrifugation, this is not necessarily the case. Centrifugation needs to be performed with sufficient g-force in appropriately calibrated and enclosed biosafe centrifuges. Smear microscopy using "direct smears" only is appropriate and sufficient for assessment of patient infectivity.

\section{Bacterial culture}

For patients with a clinical suspicion of TB (symptoms, signs and radiograph), specimens should be taken for diagnosis by microscopic examination and bacterial culture prior to the initiation of treatment. In practice taking more than three sputum specimens for the diagnosis of pulmonary $\mathrm{TB}$ is not recommended and two high-quality specimens may be sufficient. Urine samples should only be cultured where there is a clear suspicion of renal TB.

A combination of liquid (e.g. Middlebrook 7H9, Kirchner) and solid culture (e.g. Löwenstein-Jensen, Middlebrook 7H10, 7H11) gives the most optimal rates of mycobacterial recovery for diagnosis. Where resources permit, automated liquid culture systems can be used. Solid culture media will remain the backbone of culture in many countries for the foreseeable future [32-37].

Cultures for treatment monitoring (and treatment failure) should be limited in number and not performed more frequently (both in frequency and number of specimens) than indicated in international guidelines.

\section{Identification}

Positive cultures should be identified as Mycobacterium tuberculosis within 1-2 working days of receipt (ideally with the concurrent identification of rifampicin resistance, see Drug susceptibility testing). Laboratory systems should aim to culture and identify $M$. tuberculosis from sputum within 21 days of receiving the patient's specimen (and within 30 days for any pulmonary specimen) in at least $90 \%$ of cases. In practice this will mean employing liquid culture (manual or automated) and/or novel molecular diagnostic systems.

The laboratory, as well as clinicians, should recognise the risk of false-positive cultures and should assess the results in connection with clinical findings and laboratory quality assurance data.

\section{Drug susceptibility testing}

The real current, global level of drug resistance is unknown, although national and regional studies and anecdotal evidence indicate that it has been increasing in recent years [1, 3, 38, 39]. Most Western and Central European countries do not have a significant problem with drug resistance in newly diagnosed cases, reporting $\sim 5-10 \%$ isoniazid resistance and $1-2 \%$ MDRTB (resistance to at least rifampicin and isoniazid) $[1,3,39,40]$. Countries in Eastern Europe, however, have reported significantly higher rates of MDR-TB, particularly in countries of the former Soviet Union reporting rates of $\sim 10-20 \%$ in new patients [4-6, 41-46]. Previously treated patients, especially in Eastern Europe, show high rates of isoniazid resistance and MDR-TB. 
Modern four-drug treatment regimens (i.e. isoniazid, rifampicin, pyrazinamide, and ethambutol (or streptomycin)) are designed to successfully treat drug-sensitive TB and isoniazidresistant TB (with a 2-month intensive phase of treatment) [47-50]. These points form the basis of the recommendations given below.

DST is recommended for: all new TB cases; for first-line drugs with specimens taken before initiating treatment; if the patient continues to be culture positive after 2-3 months; and if there is a history of prior TB treatment (a major risk factor for drug resistance). Individual circumstances may dictate additional testing. Accuracy is more important than speed and DST results should come from a small number of well-equipped, experienced laboratories who participate and perform well in an international DST quality-control scheme. The WHO Supranational Laboratory Quality Control Network offers the greatest global coverage assessing participating laboratories in their ability to identify isoniazid, rifampicin, ethambutol and streptomycin resistance correctly [3].

The absolute concentration, resistance ratio, and proportion methods can all give accurate results provided they are carefully quality controlled and standardised [3]. For the basic principles of these methods for first-line drugs see [51, 52]. As a minimum, laboratories supplying DST data to clinicians, government, the $\mathrm{WHO}$, and for surveys or surveillance, should correctly identify resistance to isoniazid and rifampicin in $>90 \%$ of quality control samples in two out of the last three quality control rounds.

In Europe, the early identification of mycobacterial growth as M. tuberculosis complex (MTBC; principally M. tuberculosis and $M$. bovis) and the identification of rifampicin resistance should be the first priority as rifampicin resistance invalidates standard 6-month short-course chemotherapy, and is a useful marker in most countries for MDR-TB. Laboratories should aim to identify isolates as MTBC and perform rifampicin resistance in $90 \%$ of isolates within 1-2 working days. This is technologically feasible [53-67].

The early identification of isoniazid resistance in $M$. tuberculosis isolates is also of importance although less critical than rifampicin resistance. Modern molecular techniques permit the successful identification of isoniazid resistance in $\geqslant 75 \%$ of MTBC isolates within 1-2 working days and are useful preliminary screens for isoniazid resistance.

Laboratories should aim to identify $M$. tuberculosis and rifampicin resistance in $>90 \%$ of cases from smear-positive sputum directly where resources are available for this (this will require an investment in new methodological techniques). This is technologically feasible but challenging. In a recent analysis of a routine national nontrial service, 1,997 primary clinical specimens, including 658 nonrespiratory specimens were analysed [67]. The overall adjusted concordance, sensitivity, specificity, positive predictive value, and negative predictive value for detecting MTBC were 91.2, 85.2, 96.2, 95.7, and 86.7\%, respectively (unadjusted, 86.7, 85.2, 88.2, 86.9, and 86.7\%, respectively), when false-positive samples from patients $(n=83)$ with a known microbiological diagnosis of MTBC or patients receiving current or recent antituberculous treatment were excluded [67].
The nonrespiratory specimen types with the highest sensitivity rates were vertebral aspirated biopsy specimens $(n=30$, sensitivity $83.3 \%)$, gastric aspirates $(n=18$, sensitivity $80.0 \%)$ and lymph node aspirates/biopsy specimens $(n=144$, sensitivity $72.5 \%$ ). Sensitivities are significantly lower for pleural, ascitic or cerebrospinal fluid. The parameters for detecting rifampicin resistance were 99.1, 95.0, 99.6, 92.7, and 99.7\%, respectively [67].

Further operational research is needed to develop methods which can identify TB and rifampicin and isoniazid resistance, in all sputum specimens with the same sensitivity as the best bacterial culture methods, but within 1-2 working days.

For patients with MDR-TB or who are genuinely unable to tolerate first-line therapy, second-line drug therapy should be initiated. There remains a need to standardise second-line drug resistance testing and such testing should only be performed at the NRLs in Western and Central European countries due to the relatively small number of cases and the concomitant difficulty of maintaining testing proficiency if multiple centres perform this activity. It is proposed that a centre performs second-line testing only if it is performing such analyses on at least 50 new patients or 200 specimens per annum in order to maintain expertise. This argument holds for smaller Eastern European countries, such as the Baltic states, where the overall case numbers are small but additional qualified centres will be needed for the larger countries such as Russia, Ukraine and Turkey. There is a need to further develop international quality assurance for second-line drug resistance analysis.

Standardised second-line treatment programmes based on surveys or individualised treatment will produce higher treatment cure rates than no therapy or first-line drug therapy alone, although treatment must be prolonged. Although individualised treatment strategies will produce the highest cure rates, this will be dependent on the continuous availability of appropriate drugs and the adherence of the patient to the drug regimen. It is the responsibility of the laboratory, in consultation with the clinician, to determine the spectrum of drugs to be tested within the laboratory rather than the other way round.

The frequency of repeat testing of known MDR-TB cases should be limited. Whilst it is true that new resistances can emerge reasonably quickly, a greater priority is often to ensure that the reasons for a patient acquiring MDR-TB, or developing MDR-TB therapy on treatment, are identified and corrected before new drugs are administered. Clinicians should identify where they are simply determining treatment progress (i.e. they only need to establish that the patient is still smear/ culture positive) so that DST is not repeated unnecessarily. In practice, patients with established MDR-TB do not need to have the DST repeated more than every 2 months and in most cases every 6 months is sufficient.

\section{CONCLUSIONS}

The above standards are technologically feasible but may not initially be within the financial capacity of all laboratories. There is a requirement for government and international donors to adequately fund an appropriate, safe, infrastructure in which well-trained staff working to clear standard operating procedures can deliver accurate and timely results 
at whichever level of activity they are performing (microscopy, culture, drug susceptibility testing, etc.). There remains a need for national reference laboratories to train a new cadre of mycobacterial laboratory experts. This will require the funding of appropriate individuals at these centres to train and assist in the implementation of good laboratory practice and evaluation in the field in order to build sustainable capacity. Further operational research is needed to establish the optimal configuration of new technologies to determine isoniazid, rifampicin and second-line drug susceptibility in mycobacterial cultures and, increasingly, directly on specimens. Despite the pessimistic statements found in many textbooks, it is currently feasible to diagnose nearly all patients with infectious pulmonary tuberculosis and rifampicin resistance, and most with isoniazid resistance, within 1-2 working days. Improved integration of laboratory medicine as a core part of all tuberculosis programmes is needed to achieve and maximise the potential of new technological developments.

\section{ACKNOWLEDGEMENTS}

The Task Force would like to thank all the microbiologists who commented on the guidelines.

This manuscript was drafted on behalf of the WHO Euro Laboratory Strengthening Task Force (F.A. Drobniewski; S. Hoffner; S. Rusch-Gerdes; V. Thomsen; R. Zaleskis; G. Skenders; V. Malakov; M. Aziz; Y. Yurasova; D. Zalloco; V. Katalinic-Jankovic; and L. Ditiu), and approved by the WHO Euro Technical Advisory Group.

\section{REFERENCES}

1 Global tuberculosis control: surveillance, planning, financing. WHO report 2006. WHO/HTM/TB/2006.362. Geneva, World Health Organization, 2006.

2 Dye C, Scheele S, Dolin P, Pathania V, Raviglione MC. Consensus statement - global burden of tuberculosis: estimated incidence, prevalance, and mortality by country. WHO Global Surveillance and Monitoring Project. JAMA 1999; 282: 677-686.

3 World Heath Organisation. Anti-tuberculosis drug resistance in the world: third global report/the WHO/IUATLD global project on anti-tuberculosis drug resistance surveillance, 1999-2002. WHO/HTM/TB/2004.343. Geneva, World Health Organistion.

4 Drobniewski FA, Balabanova Y, Ruddy M, et al. Rifampinand multidrug-resistant tuberculosis in Russian civilians and prison inmates: dominance of the Beijing strain family. Emerg Infect Dis 2002; 8: 1320-1326.

5 Drobniewski FA, Balabanova Y, Nikolayevsky V, et al. Drug resistant tuberculosis, clinical virulence and the dominance of the Beijing strain family in Russia. JAMA 2005; 293: 2726-2731.

6 Toungoussova OS, Sandven P, Mariandyshev AO, Nizovtseva NI, Bjune G, Caugant DA. Spread of drugresistant Mycobacterium tuberculosis strains of the Beijing genotype in the Archangel Oblast, Russia. J Clin Microbiol 2002; 40: 1930-1937.

7 American Thoracic Society, Centers for Disease Control and Prevention, Infectious Diseases Society of America. American Thoracic Society/Centers for Disease Control and Prevention/Infectious Disease Society of America: controlling tuberculosis in the United States. Am J Respir Crit Care Med 2005; 172: 1169-1227.

8 American Thoracic Society, Centers for Disease Control, Council of the Infectious Disease Society of America. Diagnostic standards and classification of tuberculosis in adults and children. Am J Respir Crit Care Med 2000; 161: 1376-1395.

9 Association of Public Health Laboratories. The future of TB laboratory services-a framework for integration, collaboration, leadership. Washington, DC, Association of Public Health Laboratories, 2004.

10 Drobniewski FA, Caws M, Gibson A, Young D. Modern laboratory diagnosis of tuberculosis. Lancet Infect Dis 200, 3: 141-147.

11 Migliori GB, Raviglione MC, Schaberg T, et al. Tuberculosis management in Europe. Task Force of the European Respiratory Society (ERS), the World Health Organisation (WHO) and the International Union against Tuberculosis and Lung Disease (IUATLD) Europe Region. Eur Respir J 1999; 14: 978-992.

12 National Collaborating Centre for Chronic Conditions. Tuberculosis: clinical diagnosis and management of tuberculosis, and measures for its prevention and control. London, Royal College of Physicians, 2006.

13 National Tuberculosis Advisory Committee. Guidelines for Australian mycobacteriology laboratories. Commun Dis Intell 2006; 30: 116-128.

14 Joint Tuberculosis Committee of the British Thoracic Society. Chemotherapy and management of tuberculosis in the United Kingdom: recommendations 1998. Thorax 1998; 53: 536-548.

15 Pozniak AL, Miller RF, Lipman MC, et al. BHIVA treatment guidelines for tuberculosis (TB)/HIV infection 2005. HIV Med 2005; 6: Suppl. 2, 62-83.

16 Schwoebel V, Lambregts-Van Weez CSB, Moro ML, et al. Standardisation of tuberculosis drug resistance surveillance in Europe. Recommendations of a World Health Organization (WHO) and International Union Against Tuberculosis and Lung Disease (IUATLD) Working Group. Eur Respir J 20001; 16: 364-371.

17 Shinnick TM, Iademarco MF, Ridderhof JC. National plan for reliable tuberculosis laboratory services using a systems approach. Recommendations from CDC and the Association of Public Health Laboratories Task Force on Tuberculosis Laboratory Services. MMWR Recomm Rep 2005; 54: 1-12.

18 World Health Organisation. Guidelines for the programmatic management of drug-resistant tuberculosis. WHO/ $\mathrm{HTM} / \mathrm{tb} / 2006.361$. www.who.int/tb. Geneva.

19 Tuberculosis Coalition for Technical Assistance. International Standards for Tuberculosis Care (ISTC). The Hague, Tuberculosis Coalition for Technical Assistance, 2006.

20 Tuberculosis Division International Union Against Tuberculosis and Lung Disease. Tuberculosis bacteriologypriorities and indications in high prevalence countries: position of the technical staff of the Tuberculosis Division of the International Union Against Tuberculosis and Lung Disease. Int J Tuberc Lung Dis 2005; 9: 355-361. 
21 World Health Organisation Guidelines for the Programmatic Management of Drug-resistant Tuberculosis. Geneva, Switzerland, 2006.

22 Woods GL, Long TA, Witebsky FG. Mycobacterial testing in clinical laboratories that participate in the College of American Pathologists Mycobacteriology Surveys: changes in practices based on responses to 1992, 1993, and 1995 questionnaires. Arch Pathol Lab Med 1996; 120: 429-435.

23 Richmond JY, Knudsen RC, Good RC. Biosafety in the clinical mycobacteriology laboratory. Clin Lab Med 1996; 16: 527-550.

24 Centers for Disease Control and Prevention. Guidelines for preventing the transmission of Mycobacterium tuberculosis in health-care settings - 2005. MMWR 2005; 54: 1-147.

25 Advisory Committee on Dangerous Pathogens UK Health and Safety Executive. The management, design and operation of microbiological containment laboratories. London, UK, 2001.

26 Health and Safety Executive. Health Services Advisory Committee. Safe working and the prevention of infection in clinical laboratories and similar facilities. London, UK, 2003.

27 Centers for Disease Control and Prevention. National Institutes of Health. Biosafety in microbiological and biomedical laboratories. HHS Publication No. (CDC) 998395. www.cdc.gov/od/ohs/biosfty/bmbl4/bmbl4toc.htm. Washington, US Government Printing Office, 1999.

28 Ward-Cook K. Medical laboratory workforce trends and projections: what is past is prologue. Clin Leadersh Manag Rev 2002; 16: 364-369.

29 Figueroa-Munoz J, Palmer K, Poz MR, Blanc L, Bergstrom K, Raviglione M. The health workforce crisis in TB control: a report from high-burden countries. Hum Resour Health 2005; 3: 2.

30 Pascopella L, Kellam S, Ridderhof J, et al. Laboratory reporting of tuberculosis test results and patient treatment initiation in California. J Clin Microbiol 2004; 42: 4209-4213.

31 Ba F, Rieder HL. A comparison of fluorescence microscopy with the Ziehl-Neelsen technique in the examination of sputum for acid-fast bacilli. Int J Tuberc Lung Dis 1999; 3: 1101-1105.

32 Alcaide F, Benitez MA, Escriba JM, Martin R. Evaluation of the BACTEC MGIT 960 and the MB/BacT systems for recovery of mycobacteria from clinical specimens and for species identification by DNA AccuProbe. J Clin Microbiol 2000; 38: 398-401.

33 Brunello F, Favari F, Fontana R. Comparison of the MB/ BacT and BACTEC 460 TB systems for recovery of mycobacteria from various clinical specimens. J Clin Microbiol 1999; 37: 1206-1209.

34 Hanna BA, Ebrahimzadeh A, Elliott LB, et al. Multicenter evaluation of the BACTEC MGIT 960 system for recovery of mycobacteria. J Clin Microbiol 1999; 37: 748-752.

35 Piersimoni C, Scarparo C, Callegaro A, et al. Comparison of $\mathrm{MB} /$ Bact alert 3D system with radiometric BACTEC system and Lowenstein-Jensen medium for recovery and identification of mycobacteria from clinical specimens: a multicenter study. J Clin Microbiol 2001; 39: 651-657.

36 Pfyffer GE, Welscher HM, Kissling P, et al. Comparison of the Mycobacteria Growth Indicator Tube (MGIT) with radiometric and solid culture for recovery of acid-fast bacilli. J Clin Microbiol 1997; 35: 364-368.

37 Tortoli E, Cichero P, Piersimoni C, Simonetti MT, Gesu G, Nista D. Use of BACTEC MGIT 960 for recovery of mycobacteria from clinical specimens: multicenter study. $J$ Clin Microbiol 1999; 37: 3578-3582.

38 Dye C, Espinal MA, Watt CJ, Mbiaga C, Williams BG. Worldwide incidence of multidrug-resistant tuberculosis. J Infect Dis 2002; 185: 1197-1202.

39 World Health Organisation. Anti-tuberculosis drug resistance in the world. The WHO/IUATLD Global Project on anti-tuberculosis drug resistance surveillance. Geneva, Switzerland, 1997.

40 Irish C, Herbert J, Bennett D, et al. Database study of antibiotic resistant tuberculosis in the United Kingdom, 1994-6. BMJ 1999; 318: 497-498.

41 Dewan P, Sosnovskaja A, Thomsen V, et al. High prevalence of drug-resistant tuberculosis, Republic of Lithuania, 2002. Int I Tuberc Lung Dis 2005; 9: 170-174.

42 Krüüner A, Hoffner SE, Sillastu H, et al. Spread of drugresistant pulmonary tuberculosis in Estonia. I Clin Microbiol 2001; 39: 3339-3345.

43 Skenders G. Multidrug-resistant tuberculosis detection, Latvia. Emerg Infect Dis 2005; 11: 1461-1463.

44 Mokrousov I, Otten T, Vyazovaya A, et al. PCR-based methodology for detecting multidrug-resistant strains of Mycobacterium tuberculosis Beijing family circulating in Russia. Eur J Clin Microbiol Infect Dis 2003; 22: 342-348.

45 Ruddy M, Y Balabanova Y, Graham C, et al. Rates of drug resistance and risk factor analysis in civilian and prison patients with tuberculosis in Samara Region, Russia. Thorax 2005; 60: 130-135.

46 Kubica T, Agzamova R, Wright A, et al. The Beijing genotype is a major cause of drug-resistant tuberculosis in Kazakhstan. Int J Tuberc Lung Dis 2005; 9: 646-653.

47 Controlled clinical trial of short-course ( 6 month) regimens of chemotherapy for treatment of pulmonary tuberculosis. Lancet 1972; 1; 1079-1084.

48 Controlled trial of four 6-month regimens of chemotherapy for pulmonary tuberculosis, Second report. Second East African/British Medical Research Council Study. Am Rev Respir Dis 1976; 114: 471-475.

49 Combs DL, O'Brien RJ, Geiter LJ. USPHS Tuberculosis Short-Course Chemotherapy Trial 21: effectiveness, toxicity, and acceptability. The report of final results. Ann Intern Med 1990; 112: 397-406.

50 Jindani A, Nunn AJ, Enarson DA. Two 8-month regimens of chemotherapy for treatment of newly diagnosed pulmonary tuberculosis: international multicentre randomised trial. Lancet 2004; 364: 1244-1251.

51 Canetti G, Froman S, Grosset J, et al. Mycobacteria: laboratory methods for testing drug sensitivity and resistance. Bull World Health Organ 1963; 29: 565-578.

52 Canetti G, Fox W, Khomenko A, et al. Advances in techniques of testing mycobacterial drug sensitivity, and the use of sensitivity tests in tuberculosis control programmes. Bull World Health Organ 1969; 41: 21-43.

53 Gingeras TR, Ghandour G, Wang E, et al. Simulataneous genotyping and species identification using hybridisation pattern recognition analysis of generic Mycobacterium DNA arrays. Genome Research 1998; 8: 435-448. 
54 Saiki RK, Walsh PS, Levenson $\mathrm{CH}$, Erlich HA. Genetic analysis of amplified DNA with immobilized sequence specific oligonucleotide probes. Proc Natl Acad Sci USA 1989; 86: 6230-6234.

55 Watterson SA, Wilson SM, Yates MD, Drobniewski FA. Comparison of three molecular assays for rapid detection of rifampicin resistance in Mycobacterium tuberculosis. J Clin Microbiol 1998; 36: 1969-1673.

56 De Beenhouwer $H$, Lhiang Z, Jannes G, et al. Rapid detection of rifampicin resistance in sputum and biopsy specimens from tuberculosis patients by PCR and line probe assay. Tuberc Lung Dis 1995; 76: 425-430.

57 Drobniewski FA, Watterson SA, Wilson SM, Harris GS. A clinical, microbiological and economic analysis of a national UK service for the rapid molecular diagnosis of tuberculosis and rifampicin resistance in Mycobacterium tuberculosis. J Med Microbiol 2000; 49: 271-278.

58 Mokrousov I, Filliol I, Legrand E, et al. Molecular characterization of multiple-drug-resistant Mycobacterium tuberculosis isolates from northwestern Russia and analysis of rifampin resistance using RNA/RNA mismatch analysis as compared to the line probe assay and sequencing of the rpoB gene. Res Microbiol 2002; 153: 213-219.

59 Nikolayevsky V, Brown T, Balabanova Y, Ruddy M, Fedorin I, Drobniewski F. Detection of mutations associated with isoniazid and rifampin resistance in Mycobacterium tuberculosis isolates from Samara Region, Russian Federation. J Clin Microbiol 2004; 42: 4498-4502.

60 Troesch A, Nguyen H, Miyada CG, et al. Mycobacterium species identification and rifampin resistance testing with high-density DNA probe arrays. J Clin Microbiol 1999; 37: 49-55.

61 Eltringham IJ, Drobniewski FA, Mangan JA, Butcher PD, Wilson SM. Evaluation of reverse transcription-PCR and a bacteriophage-based assay for rapid phenotypic detection of rifampin resistance in clinical isolates of Mycobacterium tuberculosis. J Clin Microbiol 1999; 37: 3524-3527.

62 El-Hajj HH, Marras SA, Tyagi S, Kramer FR, Alland D. Detection of rifampin resistance in Mycobacterium tuberculosis in a single tube with molecular beacons. J Clin Microbiol 2001; 39: 4131-4137.

63 Piatek AS, Tyagi S, Pol AC, et al. Molecular beacon sequence analysis for detecting drug resistance in Mycobacterium tuberculosis. Nat Biotechnol 1998; 16: 359-363.

64 Riska PF, Jacobs WR. The use of luciferase-reporter phage for antibiotic - susceptibility testing in mycobacteria. Methods Microbiol 1998; 101: 431-455.

65 Wilson SM, Al-Suwaidi Z, McNerney R, Porter J, Drobniewski FA. Evaluation of a new rapid bacteriophagebased method for the drug susceptibility testing Mycobacterium tuberculosis. Nature Medicine 1997; 3: 465-468.

66 Albert H, Trollip AP, Mole RJ, Hatch SJ, Blumberg L. Rapid indication of multidrug-resistant tuberculosis from liquid cultures using FASTPlaque TB-RIF, a manual phagebased test. Int J Tuberc Lung Dis 2002; 6: 523-528.

67 Sam IC, Drobniewski F, More P, Kemp M, Brown T. Mycobacterium tuberculosis and rifampin resistance, United Kingdom. Emerg Infect Dis 2006; 12: 752-759. 